\title{
Some cautions regarding statistical power in split-plot designs
}

\author{
DRAKE R. BRADLEY and RONALD L. RUSSELL \\ Bates College, Lewiston, Maine
}

\begin{abstract}
We show that if overall sample size and effect size are held constant, the power of the $F$ test for a one-way analysis of variance decreases dramatically as the number of groups increases. This reduction in power is even greater when the groups added to the design do not produce treatment effects. If a second independent variable is added to the design, either a split-plot or a completely randomized design may be employed. For the split-plot design, we show that the power of the $F$ test on the betweengroups factor decreases as the correlation across the levels of the within-groups factor increases. The attenuation in between-groups power becomes more pronounced as the number of levels of the withingroups factor increases. Sample size and total cost calculations are required to determine whether the split-plot or completely randomized design is more efficient in a particular application. The outcome hinges on the cost of obtaining (or recruiting) a single subject relative to the cost of obtaining a single observation: We call this the subject-to-observation cost (SOC) ratio. Split-plot designs are less costly than completely randomized designs only when the SOC ratio is high, the correlation across the levels of the within-groups factor is low, and the number of such levels is small.
\end{abstract}

Most researchers know that the power of the $F$ test in the analysis of variance is a decreasing function of experimental error $\left(\sigma_{\varepsilon}\right)$ and an increasing function of sample size $(n)$, treatment effects $\left(\tau_{j}\right)$, and significance level $(\alpha)$. In this paper, we examine several aspects of experimental design that also have an impact on statistical power but that are less well known. We consider first the effect on power of increasing the number of groups in a oneway design while holding the total number of observations in the experiment constant. In one scenario, the groups added to the design preserve the overall effect size, whereas, in another, they gradually reduce the effect size, thus simulating the addition of "control" groups. Next, we consider the power and sample size requirements for the $F$ test on the between-groups factor in a split-plot factorial design. In particular, we examine how power and required sample size vary as a function of the correlation and the number of levels of the within-groups factor. We then illustrate a simple procedure for estimating the total cost of a split-plot design (as compared with a completely randomized design) that employs a sample size large enough to maintain comparable power on the

The authors are greatly indebted to Charles Reeve for supplying FORTRAN subroutines for computing the $C D F$ of the noncentral $F$ distribution. These routines were translated and incorporated into DATASIM 1.2, the software package used to compute power and sample size for the present paper. The authors also wish to acknowledge the valuable assistance of the reviewers: Richard Lehman, Joseph Rossi, and an anonymous reviewer. J. Rossi's comments, in particular, were critical in shaping the revisions of an initial draft of the manuscript. Any errors that exist in the paper are, of course, the responsibility of the authors. Correspondence should be addressed to D. R. Bradley, Department of Psychology, Bates College, Lewiston, ME 04240 (e-mail: dbradley@) abacus.bates.edu). between-groups factor. Finally, we present several guidelines that researchers can use to decide whether to employ a spit-plot or completely randomized design.

\section{Power in One-Way Experimental Designs}

To compute power for a one-way design, the researcher must first determine the value of the noncentrality parameter, $\lambda$. A convenient formula to use for this purpose is $\lambda=n k f^{2}=N f^{2}$, where $n$ is the sample size per condition, $k$ is the number of conditions, $N=n k$ is the total number of observations in the experiment, and $f$ is a measure of the size of the typical treatment effect relative to the standard deviation. The effect size measure for a one-way design is

$$
f=\sigma_{\tau} / \sigma_{\varepsilon}=\sqrt{\Sigma \tau_{j}^{2} / k \sigma_{\varepsilon}^{2}}=\sqrt{\Sigma\left(\mu_{j}-\mu\right)^{2} / k \sigma_{\varepsilon}^{2}} .
$$

Although the values of $\mu_{j}$ and $\sigma_{\varepsilon}^{2}$ are usually unknown, the researcher can conceptualize $f$ in terms of a varianceexplained measure such as $\eta^{2}$ (Cohen, 1988, pp. 281284). Since $f=\sqrt{\eta^{2} /\left(1-\eta^{2}\right)}$, power can be computed for effects corresponding to particular values of $\eta^{2}$. Indeed, many researchers simply employ Cohen's (1988, pp. 284-288) operational definitions of small $(f=.10$, $\left.\eta^{2}=.01\right)$, medium $\left(f=.25, \eta^{2}=.06\right)$, and large $(f=$ $.40, \eta^{2}=.14$ ) effects as guidelines for power and sample size determination. As noted by Rossi (1990), surveys of the magnitude of effects found in social psychology (Cooper \& Findley, 1982) and counseling psychology (Haase, Waechter, \& Solomon, 1982) indicate that the typical effect size in these areas corresponds to Cohen's (1988) definition of a medium effect size. It is therefore customary to perform power and sample size computations with the goal of ensuring $1-\beta=.80$ for effects of 
$f=.25$. We follow this (somewhat arbitrary) convention throughout this paper.

The power of the $F$ test is determined by the degrees of freedom ( $d f \mathrm{~s}$ ) of the $F$ ratio $\left(v_{1}\right.$ and $\left.v_{2}\right)$, the value of the noncentrality parameter $(\lambda)$, and the level of significance $(\alpha)$. Many factors contribute to the values of these parameters, some directly and some indirectly. Researchers are generally cognizant of how changes in $n, \tau_{j}, \alpha$, and $\sigma_{\varepsilon}$ affect the power of the $F$ test, but many are unaware that reallocating the total number of subjects $(N)$ available to participate in an experiment to a larger number of groups $(k)$ will reduce the power of the study, sometimes dramatically, even though the effect size remains constant. To illustrate, suppose that we want to conduct a simple two-group experiment. Sample size tables in Cohen (1988, p. 384 , Table 8.4.4) show that to ensure $1-\beta \approx .80$ for $f=.25$, we need to employ a sample size of $n=64$ subjects per group, or $N=128$ subjects overall. ${ }^{1}$ The corresponding value of the noncentrality parameter is $\lambda=$ $N f^{2}=128(.25)^{2}=8$. Now, assume that $N=128$ is in fact the size of the available subject pool-say, an introductory psychology class-or alternatively, assume that we have research funds for running exactly 128 subjects in the experiment, but no more. Given this constraint, we now wish to add several conditions to the experiment and redistribute the $N=128$ subjects among them accordingly. Before doing so, it would be wise to examine the impact on power of this apparently innocuous change from $k=2$ to $k>2$. Let us do this for two distinct scenarios: In Case 1, the experimental conditions that we add have, on average, the same size treatment effects as those already included in the experiment, so that, regardless of $k$, the effect size remains constant at $f=.25$; in Case 2, the experimental conditions that we add have means that are equal to the grand mean of the original two conditions in the experiment and therefore do not contribute additional treatment effects (i.e., $\tau_{j}=0$, for all such groups). ${ }^{2}$

Most researchers would predict little change in power for Case 1 because the effect size remains constant for all $k \mathrm{~s}$. They might predict moderate decreases in power for Case 2, however, because the overall treatment effects are being "diluted" by the inclusion of groups that produce no treatment effects at all. In fact, both of these predictions are wrong: As we will see, increases in $k$ produce dramatic reductions in power for Case 1 and produce exceptionally strong, not moderate, reductions in power for Case 2. The only factor that can account for a reduction in power for Case 1 is the change in the relative values of the numerator and denominator $d f$ of the $F$ ratio, $v_{1}=$ $k-1$, and $v_{2}=N-k$. For Case 2 , we have the additional fact that adding groups with $\tau_{j}=0$ causes the overall effect size to decrease steadily as $k$ increases: Given an initial effect size of $f=.25$ in a design with two groups, larger values of $k$ will attenuate the effect size such that $f^{\prime}=f \sqrt{2 / k}=.25 \sqrt{2 / k}$. To assess the impact of altering the relative values of $v_{1}$ and $v_{2}$ and the impact of decreasing $f$, we computed the power of the $F$ test for the Case 1 and Case 2 scenarios for $k=2$ to $k=10$, holding overall sample size constant at $N=128 .{ }^{3}$ The results are presented in the top half of Table 1 (power calculations). For Case 1, power drops from .80 with two groups to $.64, .54$, .47 , and .42 for experiments with $4,6,8$, and 10 groups, respectively. Hence, a substantial reduction in power occurs when conditions are added to an experiment, despite the fact that the conditions preserve the initial effect size. ${ }^{4}$ Furthermore, if groups are added to the experiment that do not produce treatment effects, as in Case 2, the reduction in power is even greater: Power drops from .80 in the two-group design to $.35, .19, .13$, and .10 for experiments with 4, 6, 8, and 10 groups, respectively. Adding just one such group to a two-group design causes the power to drop to .52 . This certainly should give researchers some pause for concern.

Table 1 (top) shows that if $N$ is kept constant, the power of the test decreases as $k$ increases. Of course, there is no necessary reason to hold $N$ constant other than the exigencies of limited resources. Suppose we remove the constraint that the study be conducted with only $N=128$ subjects. If we now keep the sample size per group constant at $n=64$ and increase $k$ from 2 to 10 , the overall sample size goes from 128 to 640 , the noncentrality parameter from $\lambda=8$ to $\lambda=N f^{2}=640(.25)^{2}=40$, and the power of the test from $.8015(1,126 d f)$ to $.9984(9,630 d f)$. Keeping $n$ constant as $k$ increases therefore causes power to go up, and we end up expending more resources than necessary. In order to maintain constant power, we need to employ the sample size that allows us to detect the effect of interest at the designated power, $1-\beta \approx .80$. With this approach, neither $n$ nor $N$ remains constant as $k$ increases. This is illustrated in the bottom half of Table 1 (sample size calculations), which presents the required sample size per group $(n)$ and the overall sample size for the experiment $(N)$ as a function of $k .^{5}$ For Case 1, we see that while the sample size per group decreases from 64 to 26 as $k$ goes from 2 to 10 , the overall sample size increases from 128 to 259 . Adding one group to the original two-group experiment requires an increase of $23 \%$ in overall sample size (from 128 to 157 ), and going from two to five groups requires an increase of $53 \%$ ( $128 \mathrm{vs}$. 196). These increases in sample size are necessary to offset the reduction in power that would otherwise occur with increases in numerator $d f\left(v_{1}\right)$ for constant $f$ and $N$. For Case 2, the situation is much worse, because increases in $k$ are also accompanied by reductions in the overall effect size. Consequently, as $k$ goes from 2 to 10 , the sample size per group increases from 64 to 126 , and the overall sample size increases from 128 to 1,260 . Adding one group to the original two-group experiment now requires an increase of $83 \%$ in overall sample size (from 128 to 234 ), and going from two to five groups requires an increase of $277 \%$ (128 vs. 482 ).

For the Case 1 scenario, the increased cost and effort required for an experiment with a larger $k$ may, in many instances, be adequately justified by the additional information gained. This is less likely to be true for the Case 2 
Table 1

Power and Sample Size as a Function of the Number of Groups $(k)$ in a One-Way Experimental Design

\begin{tabular}{|c|c|c|c|c|c|c|c|c|}
\hline \multirow[b]{3}{*}{$k$} & \multirow[b]{3}{*}{$v_{1}$} & \multirow[b]{3}{*}{$v_{2}$} & \multicolumn{6}{|c|}{ Power Calculations $(N=128)$} \\
\hline & & & \multicolumn{3}{|c|}{ Case 1} & \multicolumn{3}{|c|}{ Case 2} \\
\hline & & & $f$ & $\lambda$ & $1-\beta$ & $f^{\prime}$ & $\lambda$ & $1-\beta$ \\
\hline 2 & 1 & 126 & .25 & 8.00 & .8015 & .250 & 8.00 & .8015 \\
\hline 3 & 2 & 125 & .25 & 8.00 & .7065 & .204 & 5.33 & .5210 \\
\hline 4 & 3 & 124 & .25 & 8.00 & .6388 & .177 & 4.00 & .3482 \\
\hline 5 & 4 & 123 & .25 & 8.00 & .5858 & .158 & 3.20 & .2502 \\
\hline 6 & 5 & 122 & .25 & 8.00 & .5425 & .144 & 2.67 & .1923 \\
\hline 7 & 6 & 121 & .25 & 8.00 & .5060 & .134 & 2.29 & .1560 \\
\hline 8 & 7 & 120 & .25 & 8.00 & .4747 & .125 & 2.00 & .1321 \\
\hline 9 & 8 & 119 & .25 & 8.00 & .4475 & .118 & 1.78 & .1155 \\
\hline \multirow[t]{3}{*}{10} & 9 & 118 & .25 & 8.00 & .4235 & .112 & 1.60 & .1036 \\
\hline & & & \multicolumn{6}{|c|}{ Sample Size Calculations $(1-\beta=.80)$} \\
\hline & & & \multicolumn{3}{|c|}{ Case 1} & \multicolumn{3}{|c|}{ Case 2} \\
\hline$k$ & $v_{1}$ & & $f$ & $n$ & $N$ & $f^{\prime}$ & $n$ & $N$ \\
\hline 2 & 1 & & .25 & 64 & 128 & .250 & 64 & 128 \\
\hline 3 & 2 & & .25 & 52 & 157 & .204 & 78 & 234 \\
\hline 4 & 3 & & .25 & 45 & 178 & .177 & 88 & 353 \\
\hline 5 & 4 & & .25 & 39 & 196 & .158 & 96 & 482 \\
\hline 6 & 5 & & .25 & 35 & 211 & .144 & 104 & 621 \\
\hline 7 & 6 & & .25 & 32 & 224 & .134 & 110 & 769 \\
\hline 8 & 7 & & .25 & 30 & 237 & .125 & 116 & 925 \\
\hline 9 & 8 & & .25 & 28 & 248 & .118 & 121 & 1,089 \\
\hline 10 & 9 & & .25 & 26 & 259 & .112 & 126 & 1,260 \\
\hline
\end{tabular}

Note-Power and sample size were computed by DATASIM 1.2 (Bradley, Russell, \& Reeve, 1996). Overall sample size is held constant at $N=128$ for the power calculations, and power is held constant at $1-\beta=.80$ for the sample size calculations. Solutions are shown for two different cases: In Case 1, the effect size is held constant at $f=.25$ as $k$ increases; in Case 2, the effect size starts at $f^{\prime}=.25(k=2)$ and decreases steadily as $k$ increases, so as to simulate the addition of groups with $\tau_{j}=0$. For all calculations, $v_{1}=k-1, v_{2}=N-k$, and $\alpha=.05$.

scenario. The dramatic increases in sample size necessitated by adding groups with $\tau_{j} \approx 0$ suggest that this situation should be avoided whenever possible. Consider an experiment investigating the effects of test anxiety on exam performance. If we have a low-anxiety condition and a high-anxiety condition with treatment effects of $\tau_{1}=$ -10 and $\tau_{2}=+10$, respectively, then we might want to avoid adding a medium-anxiety condition, especially if we have reason to believe that it will produce a mean exam score midway between those of the low and high conditions, such that $\tau_{3}=0$. This is precisely the Case 2 scenario, and adding the medium-anxiety condition would require an increase in the overall sample size of $83 \%$ in order to maintain power at .80. If the goal is simply to establish the existence of an effect of test anxiety on test performance, then this can be achieved much more economically using the two-group (low vs. high) design. Of course, if the researcher's main interest is in establishing the nature of the underlying function relating test anxiety to performance, then the inclusion of a medium-anxiety condition is called for, and she or he must simply accept the additional cost associated with the larger required sample size.

The Case 1 and Case 2 scenarios in Table 1 probably span those that would actually arise in practice. In many instances, the initial conditions included in an experiment have been selected so as to maximize primary vari- ance-that is, the levels of the independent variable are either "extreme" or "optimal" values. If the experimenter subsequently decides to include additional experimental conditions, it is likely that the treatment effects $\left(\tau_{j}\right)$ associated with these conditions will be greater than 0 but less than the mean treatment effect of the conditions already in the experiment. If so, adding such conditions will reduce the effect size, $f$, and therefore the power. The resulting power and sample size values for this scenario fall in between those for Case 1 and Case 2 in Table 1. For this reason, the bottom half of Table 1 may be employed to obtain "best-case" (Case 1) and "worst-case" (Case 2) estimates of the required sample size for the augmented design.

The analysis presented here suggests that researchers should use only those conditions in an experiment that are reasonably necessary to answer the primary theoretical questions of interest. Including additional conditions for exploratory purposes, or for providing more refined control group comparisons, serves to reduce the power of the $F$ test. Most problematic is the Case 2 scenario: If the condition to be added to the experiment is likely to have a mean that falls near the grand mean of the conditions already included, the effect on power is quite substantial.

\section{Power in Two-Way Factorial Designs}

If the researcher decides to investigate the effects of a second independent variable in the same experiment, a 


$$
\begin{aligned}
& \lambda_{A}=N f_{A}^{2}=N \frac{\sigma_{\alpha}^{2}}{\sigma_{\varepsilon}^{2}}=\frac{n p q \Sigma\left(\mu_{i}-\mu\right)^{2}}{p \sigma_{\varepsilon}^{2}}=\frac{n q \Sigma\left(\mu_{i}-\mu\right)^{2}}{\sigma_{\varepsilon}^{2}}=\frac{n q \Sigma \alpha_{i}^{2}}{\sigma_{\varepsilon}^{2}} \\
& \lambda_{B}=N f_{B}^{2}=N \frac{\sigma_{\beta}^{2}}{\sigma_{\varepsilon}^{2}}=\frac{n p q \Sigma\left(\mu_{j}-\mu\right)^{2}}{q \sigma_{\varepsilon}^{2}}=\frac{n p \Sigma\left(\mu_{j}-\mu\right)^{2}}{\sigma_{\varepsilon}^{2}}=\frac{n p \Sigma \beta_{j}^{2}}{\sigma_{\varepsilon}^{2}} \\
& \lambda_{A B}=N f_{A B}^{2}=N \frac{\sigma_{\alpha \beta}^{2}}{\sigma_{\varepsilon}^{2}}=\frac{n p q \Sigma \Sigma\left(\mu_{i j}-\mu_{i}-\mu_{j}+\mu\right)^{2}}{p q \sigma_{\varepsilon}^{2}}=\frac{n \Sigma \Sigma\left(\mu_{i j}-\mu_{i}-\mu_{j}+\mu\right)^{2}}{\sigma_{\varepsilon}^{2}}=\frac{n \Sigma \Sigma \alpha \beta_{i j}^{2}}{\sigma_{\varepsilon}^{2}} .
\end{aligned}
$$

two-way factorial design is required. We will follow the usual convention of referring to the row and column factors of the design as factor $A$ and factor $B$, respectively. To illustrate power calculations for $A, B$, and the $A B$ interaction, we need to define the treatment effects and the noncentrality parameters for each of these effects. The treatment effect for level $i$ of factor $A$ is $\alpha_{i}=\mu_{i}-\mu$, the treatment effect for level $j$ of factor $B$ is $\beta_{j}=\mu_{j}-\mu$, and the interaction effect for cell $A B_{i j}$ is $\alpha \beta_{i j}=\mu_{i j}-\mu_{i}-\mu_{j}$ $+\mu$. Assuming $p$ levels of $A, q$ levels of $B, n$ observations per cell, and an overall sample size of $N=n p q$ observations, the noncentrality parameters are displayed at the top of this page. Note that the effect size squared is simply the ratio of the variance of the treatment (or interaction) effects to the variance of the experimental errors - for example, $f_{A}^{2}=\sigma_{\alpha}^{2} / \sigma_{\varepsilon}^{2}$. The noncentrality parameters can be computed solely in terms of effect size $\left(f_{A}, f_{B}\right.$, and $\left.f_{A B}\right)$ or in terms of the actual effects summed across levels $\left(\alpha_{i}\right.$, $\left.\beta_{j}, \alpha \beta_{i j}\right)$. The former approach is useful if the experimenter has no specific knowledge about the values of $\alpha_{i}$, $\beta_{j}, \alpha \beta_{i j}$, or $\sigma_{\varepsilon}^{2}$, in which case Cohen's (1988) guidelines for small, medium, and large effects (noted above) may be employed to determine the values of $f$. When the experimenter has pilot data that permit estimation of the actual treatment effects and experimental error, or when plausible values can be developed on the basis of other information (e.g., the minimum treatment effects of practical interest), then the latter approach may be used. Once the noncentrality parameters have been calculated, the power of the $F$ test for each effect can be determined.

An important consideration in the computation of power for a two-way design is the error variance $\left(\sigma_{\varepsilon}^{2}\right)$ to employ in computing the noncentrality parameter. We will distinguish two cases: the completely randomized (CR) design and the split-plot (SP) design (our terminology follows Kirk, 1995). A CR design has independent groups of subjects across the levels of both factors $(A$ and $B)$. In contrast, an SP design (Winer, Brown, \& Michels, 1991, p. 509) has independent groups of subjects across the levels of one factor (say, $A$ ) and repeated measures on the other factor (say, $B$ ). The repeated measures factor is often referred to as a within-groups factor, and the independent-groups factor is often referred to as a betweengroups factor. Most experimenters are aware that having correlated observations across the levels of factor $B$ will reduce the error variance for this factor, and that the power of tests comparing means across the levels of factor $B$ will therefore be higher than in an independentgroups design. What is not so obvious, however, is that the power of tests comparing means across the levels of factor $A$ will be less than in a design with independent groups on both factors (Bradley, 1988, p. 56). Why is this? One reason is the different $d f$ associated with the error terms. In a CR design, the denominator $d f$ for $F_{A}$ is $v_{2}=p q(n-1)$, whereas in an SP design with independent groups on factor $A$, the denominator $d f$ is only $v_{2}=$ $p(n-1)$. The more important reason, though, is the value of the error variance, $\sigma_{\tilde{\varepsilon}}^{2}$. In the CR design, the error variance for testing $A, B$, and the $A B$ interaction is simply the population variance for the dependent measure, $\sigma_{\varepsilon}^{2}=\sigma_{S / A B}^{2}=\sigma^{2}$. In the SP design, Winer (1971, p. 516) shows that the error variance for factor $A$ is $\sigma_{\varepsilon}^{2}=$ $\sigma_{S / A}^{2}=\sigma^{2}[1+(q-1) \rho]$, and the error variance for factor $B$ and the $A B$ interaction is $\sigma_{\varepsilon}^{2}=\sigma_{B \times S / A}^{2}=\sigma^{2}(1-\rho)$. In the present context, $\rho$ is the correlation across the levels of factor $B$ in the population. Relative to an initial baseline of $\sigma^{2}$, as the value of $\rho$ increases, the error variance for factor $B$ decreases and the error variance for factor $A$ increases. As a result, the higher the correlation across the levels of $B$, the higher the power of tests on $B$ and the lower the power of tests on $A$. Moreover, the differences between the two grow in proportion to the number of levels of factor $B(q)$.

Now, suppose a researcher assigns independent groups of subjects to the $p$ control and experimental conditions of a between-groups factor $(A)$ and then measures the performance of these subjects across the $q$ trials of a withingroups factor $(B)$. The more important independent variable in this context is usually factor $A$, because it represents the effects of the primary variable being manipulated by the experimenter, and it is the one for which hypotheses are (usually) advanced. Unfortunately, the power of the $F$ test on $A$ is substantially degraded by the inclusion of a correlated groups factor $(B)$ in the design. To show this, consider the following formulas for the effect size of $A$, $B$, and $A B$ in a CR design:

$$
\begin{aligned}
& f_{A(\mathrm{CR})}=\frac{\sigma_{\alpha}}{\sigma} \\
& f_{B(\mathrm{CR})}=\frac{\sigma_{\beta}}{\sigma}
\end{aligned}
$$




$$
f_{A B(\mathrm{CR})}=\frac{\sigma_{\alpha \beta}}{\sigma} .
$$

If we assume for our SP design that the treatment effectsas indexed by $\sigma_{\alpha}, \sigma_{\beta}$, and $\sigma_{\alpha \beta}-$ remain the same as in the $\mathrm{CR}$ design, then the effect sizes and noncentrality parameters for the SP design are given by

$$
\begin{aligned}
& f_{A}=\frac{\sigma_{\alpha}}{\sigma_{S / A}}=\frac{\sigma_{\alpha}}{\sigma \sqrt{1+(q-1) \rho}}=\frac{f_{A(\mathrm{CR})}}{\sqrt{1+(q-1) \rho}} \\
& f_{B}=\frac{\sigma_{\beta}}{\sigma_{B \times S / A}}=\frac{\sigma_{\beta}}{\sigma \sqrt{1-\rho}}=\frac{f_{B(\mathrm{CR})}}{\sqrt{1-\rho}} \\
& f_{A B}=\frac{\sigma_{\alpha \beta}}{\sigma_{B \times S / A}}=\frac{\sigma_{\alpha \beta}}{\sigma \sqrt{1-\rho}}=\frac{f_{A B(\mathrm{CR})}}{\sqrt{1-\rho}} \\
& \lambda_{A}=N f_{A}^{2}=\frac{N f_{A(\mathrm{CR})}^{2}}{1+(q-1) \rho}=\frac{\lambda_{A(\mathrm{CR})}}{1+(q-1) \rho} \\
& \lambda_{B}=N f_{B}^{2}=\frac{N f_{B(\mathrm{CR})}^{2}}{1-\rho}=\frac{\lambda_{B(\mathrm{CR})}}{1-\rho} \\
& \lambda_{A B}=N f_{A B}^{2}=\frac{N f_{A B(\mathrm{CR})}^{2}}{1-\rho}=\frac{\lambda_{A B(\mathrm{CR})}}{1-\rho} .
\end{aligned}
$$

These formulas express the effect sizes and noncentrality parameters of the SP design in terms of the corresponding values for the CR design, and this greatly facilitates comparisons of the two designs. It is evident that the presence of correlated observations on factor $B$ serves to decrease the effect size of factor $A$ and to increase the effect size of factor $B$ (and the $A B$ interaction). As $\rho$ goes from 0 to $1, f_{A}$ goes from $f_{A(\mathrm{CR})}$ to $f_{A(\mathrm{CR})} / \vee_{q}$, whereas $f_{B}$ goes from $f_{B(\mathrm{CR})}$ to $\infty$. Over the same range, $\lambda_{A}$ goes from $\lambda_{A(\mathrm{CR})}$ to $\lambda_{A(\mathrm{CR})} / q$, and $\lambda_{B}$ goes from $\lambda_{B(\mathrm{CR})}$ to $\infty$. To compute power using the above formulas, one needs a rough estimate of $\rho$, as well as the numerator $\left(v_{1}\right)$ and the denominator $\left(v_{2}\right) d f$ s for the CR and SP designs (see bottom of this page). As illustrated below, the noncentrality parameter $(\lambda)$ is computed for a given effect size $(f)$ and overall sample size $(N)$, and the power of the test determined by referencing standard power tables (Tiku, 1967, pp. 527-537). ${ }^{6}$

\section{Power in Split-Plot Designs}

We begin by demonstrating the computation of $\mathrm{CR}$ and SP power for a $2 \times 2$ factorial design with $n=16$ observations per cell. Power is computed for $A, B$, and $A B$ assuming that each produces a medium-size effect in a completely randomized design - that is, $f_{(\mathrm{CR})}=.25$. The noncentrality parameter corresponding to a medium-size effect is $\lambda_{(\mathrm{CR})}=N f_{(\mathrm{CR})}^{2}=n p q f_{(\mathrm{CR})}^{2}=(2)(2)(16)(.25)^{2}=4$. Given these values, $C R$ power is .5032 for $F_{A}(1,60)$,
$F_{B}(1,60)$, and $F_{A B}(1,60)$. For the SP design, we assume that the treatment effects $\left(\alpha_{i}, \beta_{j}\right.$, and $\left.\alpha \beta_{i j}\right)$ remain unchanged; to find SP power, it is therefore necessary to modify the effect sizes and noncentrality parameters as follows (note that $q-1=1$ ):

$$
\begin{aligned}
& f_{A}=\frac{.25}{\sqrt{1+\rho}}, \quad \lambda_{A}=\frac{4}{1+\rho} \\
& f_{B}=f_{A B}=\frac{.25}{\sqrt{1-\rho}}, \lambda_{B}=\lambda_{A B}=\frac{4}{1-\rho} .
\end{aligned}
$$

In order to compute SP power, we must assign some value to $\rho$. Assume that the correlation across the levels of the within-groups factor $(B)$ is $\rho=.50$. In this case, the effect size for factor $A$ is $f_{A}=.25 / \sqrt{ }(1+.5)=.20412$, the noncentrality parameter is $\lambda_{A}=4 /(1+.5)=2.667$, and the power of $F_{A}(1,30)$ is $.3525 .{ }^{7}$ For $F_{B}(1,30)$ and $F_{A B}(1,30)$, we have $f_{B}=f_{A B}=.25 / \sqrt{ }(1-.5)=.3536$, $\lambda_{B}=\lambda_{A B}=4 /(1-.5)=8$, and $1-\beta=.7814$. Hence, in a $2 \times 2$ design with $n=16, F_{A}$ and $F_{B}$ have CR power of .5032 and SP power (at $\rho=.50$ ) of .3536 and .7814 , respectively. These calculations show that the increased power of factor $B$ in an SP design (due to correlated observations) is obtained at the expense of factor $A$.

To examine the differences between CR and SP power in more detail, we computed the power of $A, B$, and $A B$ for values of $\rho$ ranging from 0 to 1 in increments of .10 . All calculations were based on an initial effect size of $f=.25$ at $\rho=0$ [i.e., $f_{(\mathrm{CR})}=.25$ ] in a $2 \times 2$ design with $n=16$ per cell $(N=64$ overall). The top left panel of Figure 1 shows the results. The dashed horizontal line gives the power (.5032) of $A, B$, and $A B$ in a CR design, and the three solid curves give the power of these same effects in the SP design. Factor $A$ power is represented by small filled circles, factor $B$ by small open circles, and the $A B$ interaction by large open circles. (The curves for $B$ and $A B$ coincide for all $p \times 2$ designs, because the $F$ ratios share the same error term and numerator $d f$.) The power functions clearly show the differential impact of correlated observations on power: As $\rho$ increases from 0 to 1, the power of $B$ and $A B$ increases from .4904 to 1.000 , whereas the power of $A$ decreases from .4904 to $.2777 .{ }^{8}$

Given the formulas above for $f_{A}$ and $\lambda_{A}$ in an SP design, we can anticipate that the discrepancy in power between $F_{A}$ and $F_{B}$ will grow as the number of levels of factor $B$ grows. To verify this, we next computed power for $2 \times 4(n=10)$ and $2 \times 10(n=7)$ designs. The sample size per cell was selected so as to give an initial power of about $.40-.80$ at $\rho=0$; this allows room for the power curves to diverge as $\rho$ increases. The results are presented in the two lower panels at the left of Figure 1. As expected, the power curves for the $2 \times 10$ design show the most dramatic divergence: As $\rho$ goes from 0 to 1 , the

$$
\begin{array}{llll} 
& \multicolumn{1}{c}{F_{A}} & \multicolumn{1}{c}{F_{B}} & F_{A B} \\
\mathrm{CR}\left(v_{1}, v_{2}\right) & p-1, p q(n-1) & q-1, p q(n-1) & (p-1)(q-1), p q(n-1) \\
\operatorname{SP}\left(v_{1}, v_{2}\right) & p-1, p(n-1) & q-1, p(n-1)(q-1) & (p-1)(q-1), p(n-1)(q-1)
\end{array}
$$



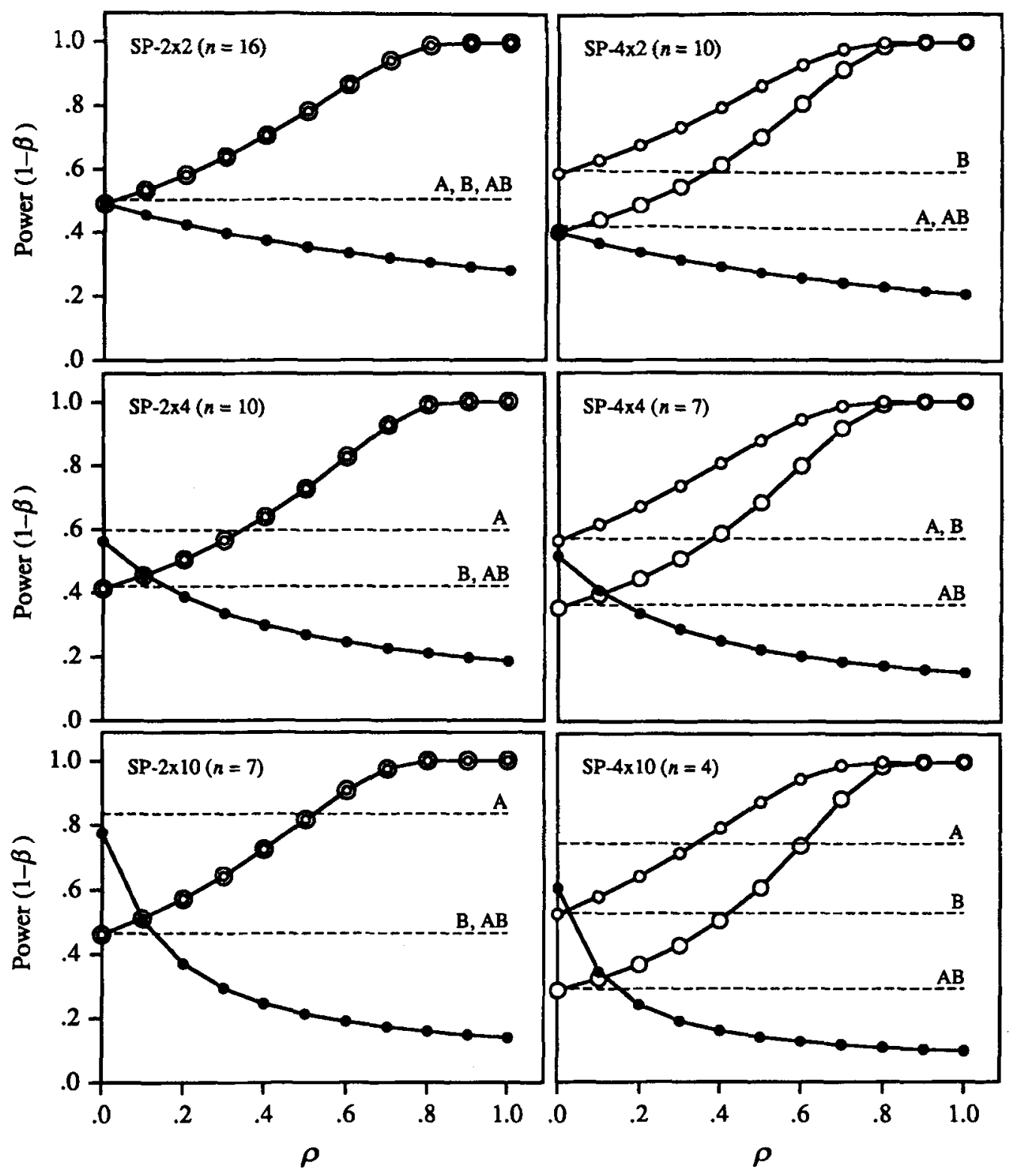

$\longrightarrow$ Power A $\longrightarrow$ Power B $\longrightarrow$ Power AB

Figure 1. The power of $F_{A}, F_{B}$, and $F_{A B}$ as a function of $\rho$ in $p \times q$ split-plot (SP) designs with correlated observations on factor $B$. Each effect has an effect size of $f=.25$ at $\rho=0$. The dashed horizontal lines show the power for $F_{A}, F_{B}$, and $F_{A B}$ in a completely randomized groups design.

power of $F_{A}$ decreases from .7750 to .1386 , whereas that of $F_{B}$ and $F_{A B}$ increases from .4606 to 1.000 . For comparison, in a $\mathrm{CR}$ design, the noncentrality parameter for $A$, $B$, and $A B$ would be $\lambda_{(\mathrm{CR})}=n p q f_{(\mathrm{CR})}^{2}=(7)(2)(10)(.25)^{2}=$ 8.75. This yields power values of 8351 for $F_{A}(1,120)$ and .4640 for $F_{B}(9,120)$ and $F_{A B}(9,120)$. These power values are plotted as dashed lines in the $2 \times 10$ panel. For the SP design, assume that $\rho=.5$, so as to parallel the example above for the $2 \times 2$ design. In this case, $\lambda_{A}=8.75 /$ $[1+(9) .5]=1.5909$, and $\lambda_{B}=\lambda_{A B}=8.75 /[1-.5]=$ 17.50. This gives power values of .2133 for $F_{A}(1,12)$ and
.8167 for $F_{B}(9,108)$ and $F_{A B}(9,108)$. These calculations, as well as visual inspection of the power curves in Figure 1 , verify that the power of $F_{A}$ deviates much more dramatically from its $(\mathrm{CR})$ baseline in the $2 \times 10$ design than in the $2 \times 2$ design. ${ }^{9}$ This is due to the effect of $q-1$ as a multiplier of $\rho$ in the denominator of $f_{A}$ and $\lambda_{A}$.

The three panels on the right of Figure 1 show the power functions for $A, B$, and $A B$ in $4 \times 2,4 \times 4$, and 4 $\times 10$ SP designs. Since factor $A$ now has more than two levels, the numerator $d f$ for the $A B$ interaction, $v_{1}=$ $(p-1)(q-1)$, differs from that of the main effect of fac- 
tor $B, v_{1}=q-1$. As a result, the power curves for $B$ and $A B$ no longer coincide. Although both rise as $\rho$ increases, reflecting the effect of the progressively smaller error variance, $\sigma_{B \times S / A}^{2}=\sigma^{2}(1-\rho)$, the power for the interaction is less than that for factor $B$ at all but the highest values of $\rho$. This is due to the larger value for the numerator $d f$ in the test on the interaction. Otherwise, the power curves for the $4 \times q$ designs confirm the findings for the $2 \times q$ designs: There is substantial attrition in the power of $F_{A}$ as $\rho$ increases, and this is most marked for large values of $q$.

\section{Sample Size Requirements for Split-Plot Designs}

Figure 1 clearly demonstrates that $\mathrm{SP}$ designs provide higher power for $B$ and $A B$, but lower power for $A$, than do CR designs. When factor $A$ is a theoretically important variable, using an SP design with repeated measures on factor $B$ would appear to be a bad idea. However, we need to consider the fact that the SP design uses fewer subjects than does the CR design. ${ }^{10}$ The total number of observations in the two designs is the same, $n p q=N=$ $N_{\text {obs }}$, but the use of repeated measures on factor $B$ means that only $n p=N_{\text {subj }}$ total subjects are required for the SP experiment, although each subject must be measured $q$ times. In the CR design, each subject is measured only once, and $n p q=N_{\text {subj }}=N_{\text {obs }}$. Consequently, the SP design has an advantage of $q: 1$ over the CR design in the number of subjects required. Since many researchers consider subject recruitment (humans) or purchase (animals) one of the major costs of doing research, the SP design is appealing precisely because it minimizes the number of subjects. However, the fact that $F_{A}$ power is less in SP designs means that we will have to increase $n$ by some amount in order to offset this loss in power. The question is, how much? Given this larger $n$, is the SP design still more efficient than a CR design?

To answer these questions, we need to determine the sample size ( $n, N_{\text {subj }}$, and $N_{\text {obs }}$ ) required by an SP design to maintain acceptable power - say, $1-\beta=.80-$ on the test of the between-groups factor $(A)$. Since the power of $B$ and $A B$ is usually higher than $A$ (Figure 1), a sample size that gives adequate power for $A$ will generally suffice to give adequate power for these effects as well. Once determined, the sample size required for the SP design can then be compared with that required for a CR design. We can anticipate certain results of these analyses. Since the power for $F_{A}$ is lower in the SP design than in the CR design, the sample size per cell $(n)$ will have to be increased accordingly to offset this loss of power. Furthermore, the higher the correlation across the levels of factor $B$ and the greater the number of levels of factor $B$, the more the sample size will have to be increased. Now, as $n$ is increased, so is the total number of subjects required by the SP design, $N_{\text {subj }}=n p$, and the main advantage of this design over the CR design begins to diminish. Also, as the sample size per cell goes up, so does the total number of observations $\left(N_{\text {obs }}\right)$ in the experiment: Each increment in $n$ by one adds $p q$ observations to $N_{\text {obs }}$. So, while the SP design uses fewer subjects $\left(N_{\text {subj }}\right)$ than the CR design, it requires more total observations $\left(N_{\mathrm{obs}}\right)$. In effect, then, the SP design exchanges effort in recruiting and acquiring subjects for effort in collecting observations. We will return to this point later when we discuss total cost analyses for SP and CR designs.

Figure 2 plots the sample sizes (as a function of $\rho$ ) required to ensure $F_{A}$ power of .80 in a $4 \times 4$ design, given $f_{A(\mathrm{CR})}=.25$. The dashed horizontal lines show the sample sizes for a CR design: The lower line indicates that $n=11$ subjects are required per cell, and the upper line indicates that $N_{\text {subj }}=N_{\text {obs }}=179$ subjects and observations are required overall. The sample size functions for the SP design are the solid lines labeled $n$ (bottom), $N_{\text {subj }}$ (middle), and $N_{\text {obs }}$ (top). As with the $4 \times 4$ power calculations in Figure 1, the SP sample size solutions were obtained after modifying the effect size measure for factor $A$ as follows:

$$
f_{A}=\frac{f_{A(\mathrm{CR})}}{\sqrt{1+(q-1) \rho}}=\frac{.25}{\sqrt{1+3 \rho}} .
$$

To illustrate, at $\rho=.50$, the effect size is $f_{A}=.1581$, and the SP design requires sample sizes of $n=28, N_{\text {subj }}=$ 113 , and $N_{\text {obs }}=452$ in order to give power of .80 for $F_{A} \cdot{ }^{11}$ We see that while the SP design requires $37 \%$ fewer subjects than the CR design (113 vs. 179), it requires $153 \%$ more total observations ( 452 vs. 179 ). As $\rho$ increases, this disparity becomes even more extreme: at $\rho=1$, the sample sizes required for the SP design are $n=45, N_{\text {subj }}=178$, and $N_{\text {obs }}=714$. In this case, the number of subjects needed is about the same as that required for a CR design, but the number of observations increases by $299 \%$. At the other extreme, when $\rho=0$, the required sample sizes are $n=12, N_{\text {subj }}=48$, and $N_{\text {obs }}=$ 191. In this event, the sample size per cell is about the same as in the CR design, the number of subjects is smaller by $73 \%$, and the total number of observations is larger by $7 \%$.

The sample size functions in Figure 2 show that the SP design loses efficiency as the correlation across the levels of the within-groups factor increases. As $\rho$ nears 1, the number of subjects required approaches that of the CR design, and the total number of observations needed is $q$ times larger than that for the $\mathrm{CR}$ design. This latter fact indicates that the relative efficiency of the SP design will vary greatly with the number of levels $(q)$ of factor $B$. To examine this, we performed sample size calculations for each of the $p \times q$ SP designs presented in Figure 1. The results are presented in Figure 3, which plots the sample sizes (as a function of $\rho$ ) required to ensure power of .80 for $F_{A}$ given $f_{A(\mathrm{CR})}=.25$. Note that the vertical axis in each plot is scaled logarithmically. Comparing the sample size functions in Figure 2 with those in the $4 \times 4$ panel of Figure 3 illustrates the effect of the logarithmic scale. In Figure 2, the ratio of $N_{\text {obs }}$ to $N_{\text {subj }}$ remains constant at $q: 1=4: 1$, and the ratio of $N_{\text {subj }}$ to $n$ remains constant at $p: 1=4: 1$, across all values of $\rho$. The slopes of the three curves in Figure 2 differ accordingly. With the logarithmic scale employed in 


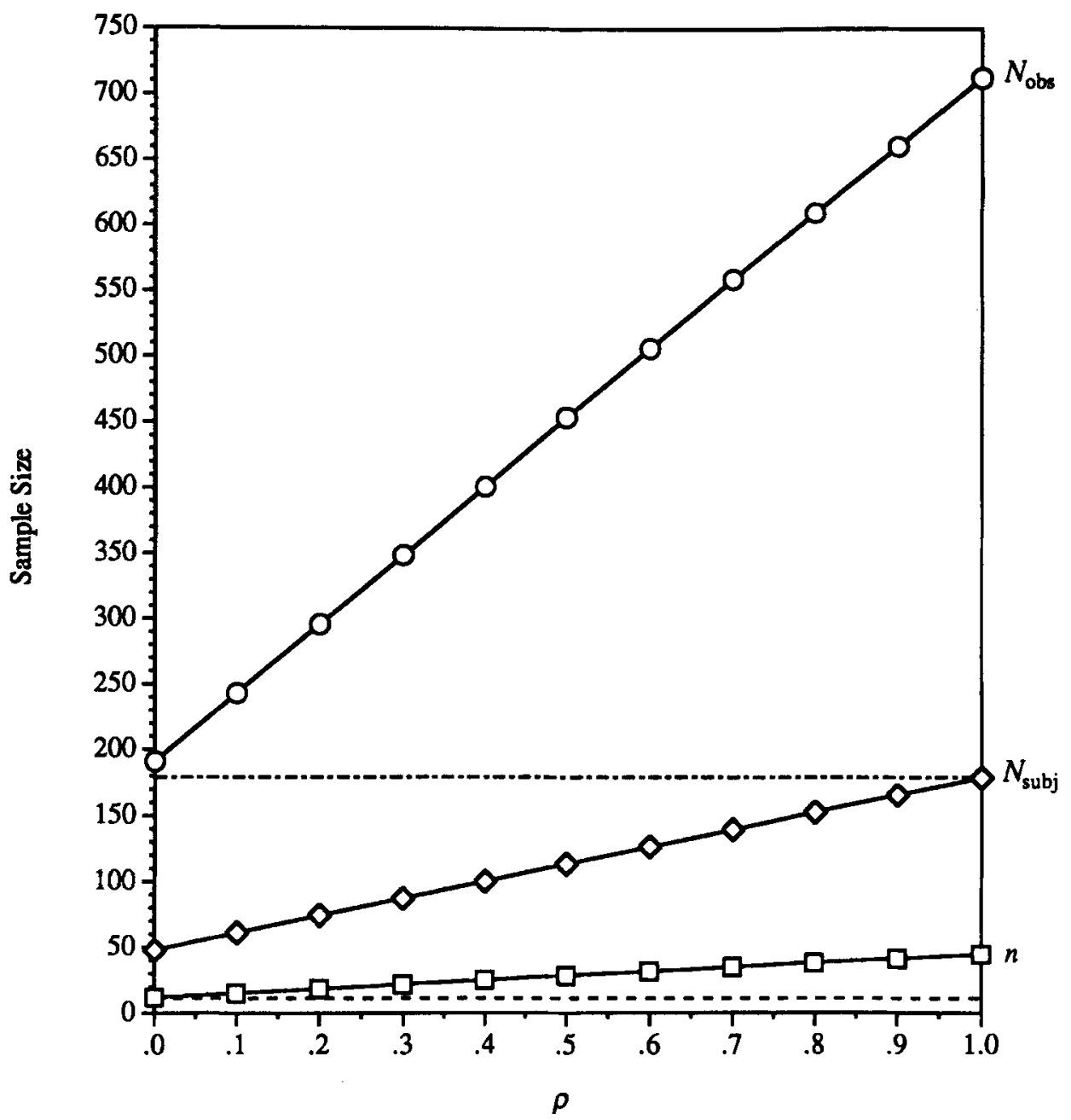

Figure 2. The sample size per cell $(n)$, number of different subjects $\left(N_{\text {subj }}\right)$, and total number of observations $\left(N_{\text {obs }}\right.$ ) required to achieve power of .80 for $F_{A}$ in a $4 \times 4$ split-plot design with correlated observations on factor $B$. Sample size is plotted as a function of $\rho$, and the initial effect size is $f=.25$ at $\rho=0$. The lower dashed horizontal gives the sample size per cell $(n)$, and the upper dashed horizontal gives both the number of subjects $\left(N_{\text {subj }}\right)$ and the total number of observations $\left(N_{\text {obs }}\right)$ required in a completely randomized groups design.

Figure 3, these same sample size functions plot as parallel lines. This prevents compression of the $n$ and $N_{\text {subj }}$ curves at the bottom of the plot for extreme ratios of $N_{\mathrm{obs}}: N_{\text {subj }}: n$, as in the $2 \times 10$ and $4 \times 10$ designs.

Consider the sample size functions for the $2 \times 10 \mathrm{de}-$ sign. For purposes of comparison, we will determine the sample size requirements for (1) a CR design and (2) an SP design with $\rho=0, .5$, and 1 . The lower dashed line in the $2 \times 10$ panel of Figure 3 indicates the required sample size $(n)$ for the CR design, and the lower solid line does the same (as a function of $\rho$ ) for the SP design. The two curves reveal that the required sample sizes are $n=6,7,36$, and 64 , respectively, for the CR design and the three SP designs ( $\rho=0, .5$, and 1$)$. The upper dashed line (CR) and the middle solid line (SP) show that the corresponding number of subjects required is $N_{\text {subj }}=$ $128,15,71$, and 128 . Finally, the upper dashed line and the upper solid line show that the required number of observations is $N_{\text {obs }}=128,148,710$, and 1,275 . We see that the SP design provides a huge savings in the total number of subjects $\left(N_{\text {subj }} \approx 15\right.$ vs. 128$)$ when the correlation is very low, but it requires an unrealistically large number of observations when the correlation is very high $\left(N_{\text {obs }} \approx 1,275\right.$ vs. 128$)$. Consider now the sample size functions for the $2 \times 2$ design. For the $C R$ design and for SP designs with $\rho=0, .5$, and 1 , the required sample sizes are: $n=32,32,48,64 ; N_{\text {subj }}=128,65,96,128$; and $N_{\text {obs }}=128,130,192,255$. Although the $2 \times 2 \mathrm{SP}$ design yields smaller savings in total subjects than does the $2 \times 10$ design at the very low correlations $\left(N_{\text {subj }} \approx 65\right.$ vs. 128), it also shows less of an increase in the total number of observations required at very high correlations $\left(N_{\mathrm{obs}} \approx 255\right.$ vs. 128$)$. Consequently, the potential benefit and cost of the SP design, relative to those of the 

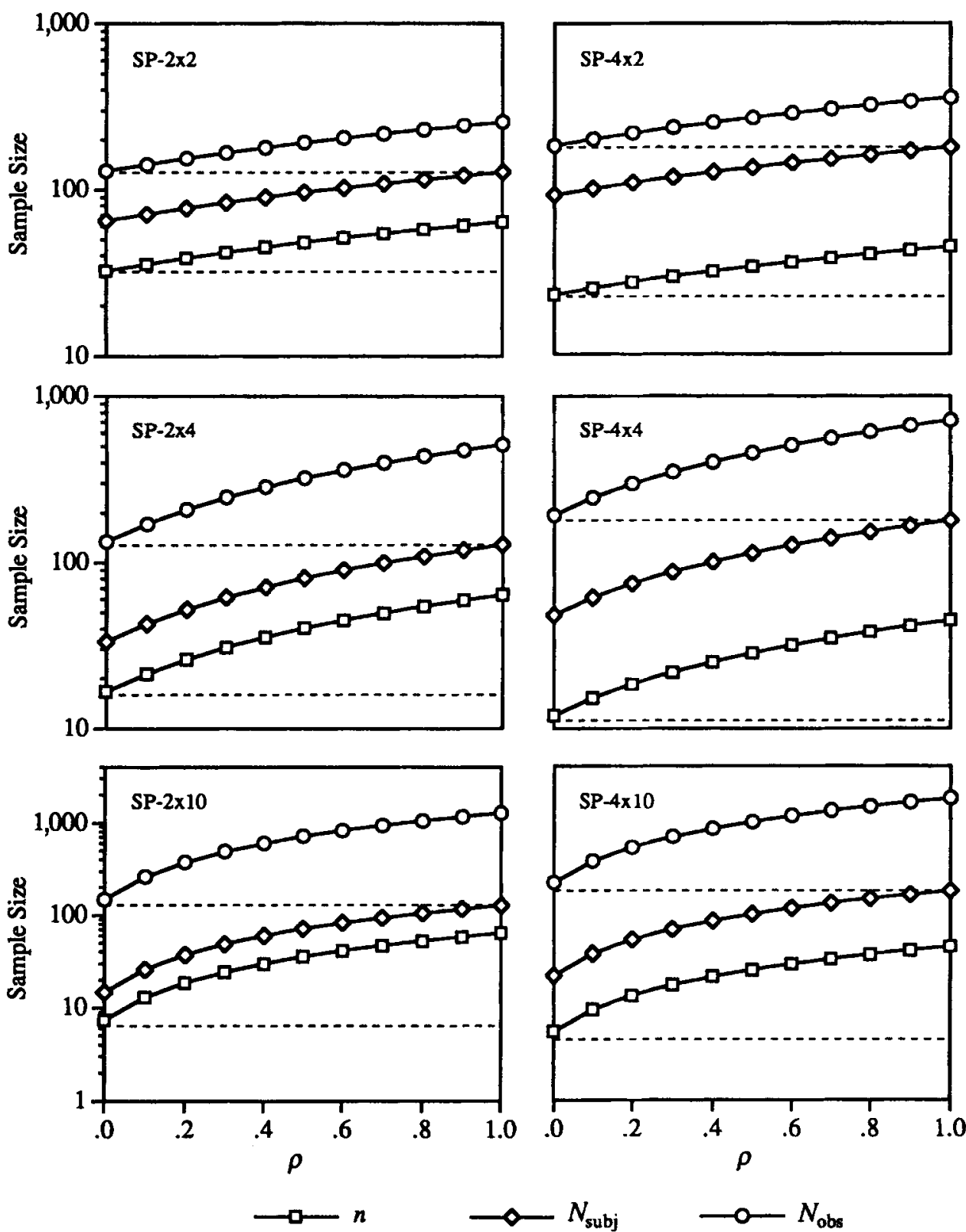

$\longrightarrow-N_{\text {obs }}$

Figure 3. The sample size per cell $(n)$, number of different subjects $\left(N_{\text {subj }}\right)$, and total number of observations $\left(N_{\text {obs }}\right)$ required to achieve power of .80 for $F_{A}$ in $p \times q$ split-plot (SP) designs with correlated observations on factor $B$. Sample size is plotted as a function of $\rho$, and the initial effect size is $f=.25$ at $\rho=0$. The lower dashed horizontal gives the sample size per cell $(n)$, and the upper dashed horizontal gives both the number of subjects $\left(N_{\text {subj }}\right)$ and the total number of observations $\left(N_{\text {obs }}\right)$ required in a completely randomized groups design.

CR design, increase with the number of levels of the repeated measures factor $(q)$.

For either sample size index, $N_{\text {subj }}$ or $N_{\text {obs }}$, we can compute the relative difference (RD) in the sample size required by an SP design as opposed to a CR design using the following formula: $R D=100(S P-C R) / C R$. Recall from above that the total number of subjects required for a $2 \times 2 \mathrm{SP}$ design is $N_{\text {subj }}=96$ if $\rho=.5$, whereas the total number of subjects required for a CR design is $N_{\text {subj }}=$
128. The relative difference in sample size is therefore $\mathrm{RD}=100(96-128) / 128=-25 \%$. In other words, the SP design requires $25 \%$ fewer subjects than the CR design in order to achieve power of .80 for detecting a medium-size effect on $F_{A}$. For the total number of observations, on the other hand, we have $N_{\mathrm{obs}}=255$ for the SP design, and $N_{\text {obs }}=128$ for the CR design. This corresponds to a relative difference of $R D=100(192-128) /$ $128=50 \%$. Thus, $50 \%$ more observations must be ob- 
tained for the SP design to yield the same power on $F_{A}$ as the CR design. Note that the reduction of $25 \%$ in total subjects is a benefit, whereas the increase of $50 \%$ in total observations is a cost. As shown below, whether the SP design results in a net benefit or cost depends on $\rho, q$, and the relative cost of obtaining subjects as opposed to observations.

Figure 4 displays the RD, expressed as a percent, in the total number of observations (top) and the total number of subjects (bottom) required by an SP design relative to a CR design. The RD in sample size is plotted as a function of $\rho$ for $2 \times 2,2 \times 4$, and $2 \times 10$ designs. ${ }^{12}$ The results show that, at high values of $\rho$, SP designs provide little or no savings in subjects, and they have very great costs in observations. At low values of $\rho$, the reverse is true: SP designs provide very great savings in subjects at relatively low cost in observations. Moreover, the potential savings in subjects (at low values of $\rho$ ) and the potential cost in observations (at high values of $\rho$ ) both in- crease with the number of levels $(q)$ of the within-groups factor. As $\rho$ goes from 0 to 1 in the $2 \times 2$ design, the savings in subjects goes from $-49 \%$ to $0 \%$, and the cost in observations goes from $1.5 \%$ to $100 \%$. In the $2 \times 10 \mathrm{de}$ sign, on the other hand, the savings in subjects goes from $-89 \%$ to $0 \%$, and the cost in observations goes from $15 \%$ to $898 \%$ over this same range. This confirms the point noted above that both $\rho$ and $q$ are critical determinants of the efficiency of the SP design relative to the CR design.

How can the experimenter determine which design to use in a particular application? For any given $q$, we need to determine the value of $\rho$ that represents the crossover point-that is, the point at which the SP design and the CR design are equally efficient. The SP design will be the more efficient design for values of $\rho$ less than the crossover, because the savings in subjects (relative to the CR design) more than offsets the increase in observations. For values of $\rho$ greater than the crossover, the SP design will be less efficient than a CR design because the (di-

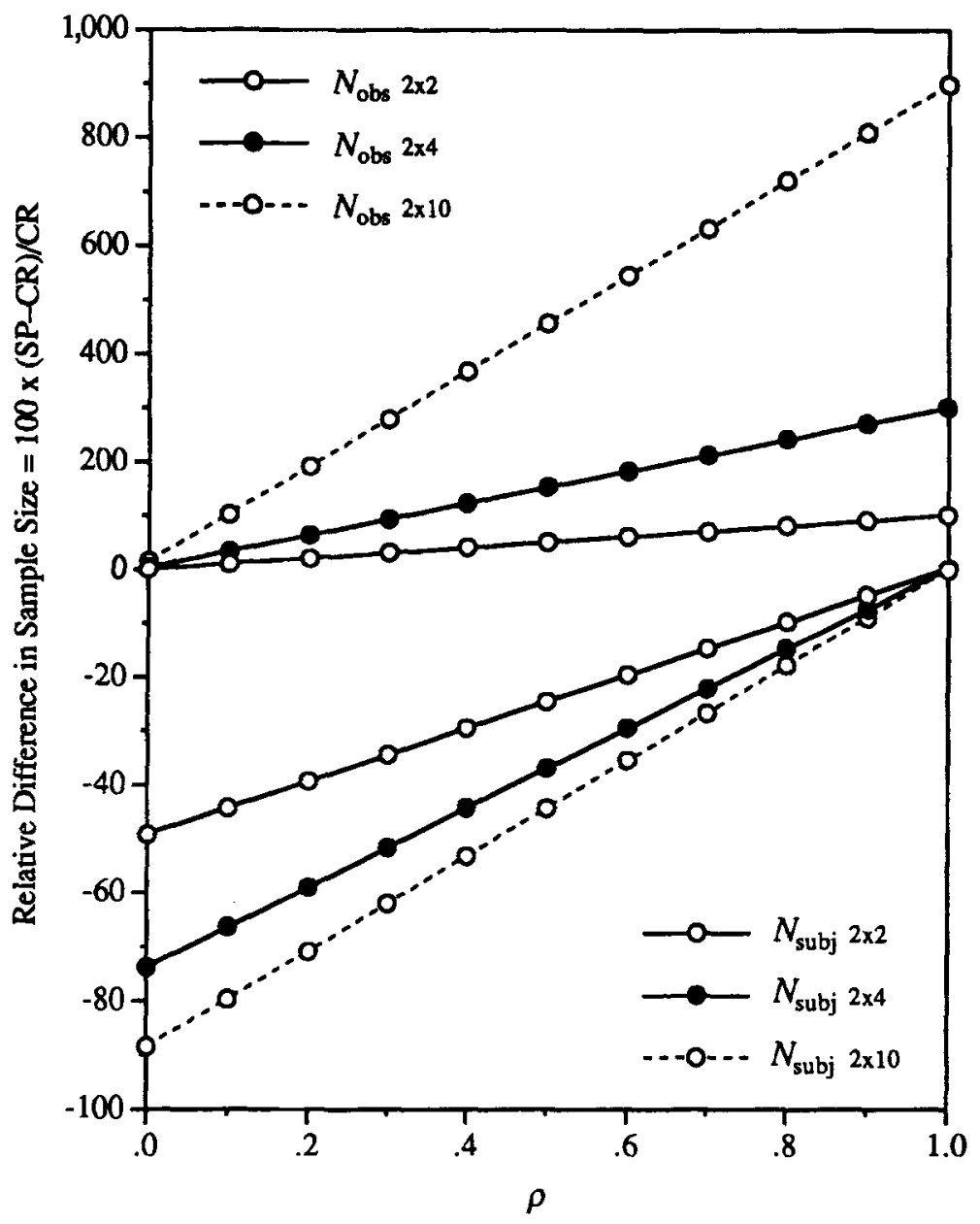

Figure 4. The percent difference in sample size ( $\boldsymbol{N}_{\text {subj }}$ and $N_{\text {obs }}$ ) required to achieve power of .80 for $F_{A}$ in $p \times q$ split-plot designs relative to completely randomized designs. 
minished) savings in subjects fails to offset the (dramatic) increase in observations. In the next section, we discuss methods for establishing the crossover point.

\section{Cost Analysis for Split-Plot Designs}

Figure 4 shows that SP designs require fewer subjects, but more observations, than CR designs. In order to make a rational choice between the two designs, it is necessary to establish some type of correspondence between the cost (and/or effort) required to obtain a subject as opposed to an observation. We will express this correspondence in the form of a ratio, called the subject-to-observation cost (SOC) ratio. For example, if the expense of obtaining a single subject is three times that of obtaining a single observation, then the SOC ratio is $3: 1$. This implies that for every $\$ 1$ expended on the experiment, $\$ 0.75$ represents the cost associated with obtaining a subject and $\$ 0.25$ represents the cost of obtaining a single observation. The SOC ratio can be established on the basis of the actual cost of these activities (in dollars), the time required to do them (in minutes), or some blend of the two. Or, if it is not convenient to estimate actual cost or effort, then the SOC ratio can simply reflect the experimenter's subjective judgment (based on past experience) of the relative weights that obtaining subjects and observations should have in the cost-benefit analysis. There is no necessary requirement that the SOC ratio reflect an actual decomposition of the costs and times involved in recruiting subjects and obtaining observations. For the analysis that follows, it is sufficient that the experimenter be able to decide that recruiting a subject is, say, roughly three times more difficult than obtaining an observation. As long as some basis exists for arriving at an SOC ratio, it is possible to determine whether the SP design or the CR design is more efficient.

We will assume for the moment that the SOC ratio reflects the relative cost (in dollars) of obtaining a subject as opposed to an observation. Once the SOC ratio is established, it is possible to determine the total cost of doing the experiment. Consider again the $2 \times 10$ design in the lower left panel of Figure 3 . The upper dashed line indicates that a CR design requires $N_{\text {subj }}=N_{\text {obs }}=128$ subjects to achieve power of .80 on $F_{A}$. To determine the total cost of the experiment, we now introduce the concept of a base dollar. Each base dollar consists of two parts: one portion represents the cost of recruiting the subject $\left(S_{\text {cost }}\right)$, and the other represents the cost of obtaining a single observation on that subject $\left(O_{\text {cost }}\right)$. If the SOC ratio is $3: 1$, then each base dollar allocates $S_{\text {cost }}=\$ 0.75$ to the cost of recruiting the subject, and $O_{\text {cost }}=\$ 0.25$ to the cost of obtaining a single observation. Assume for the moment that it costs exactly $\$ 1(\$ 0.75+\$ 0.25)$ to recruit a subject and to obtain one observation on that subject. If so, the total cost of a $2 \times 10 \mathrm{CR}$ design would be $C_{\mathrm{CR}}=$ $N_{\text {subj }}\left(S_{\text {cost }}+O_{\text {cost }}\right)=128(\$ 0.75+\$ 0.25)=\$ 128$. Since the CR design has a one-to-one correspondence between the number of subjects and the number of observations, the cost of doing the experiment is independent of the ac- tual allocation of the subject and observation costs. Hence, if the SOC ratio is $2: 1$ ( $\$ 0.67$ and $\$ 0.33$ ), the total cost is still $\$ 128$. The cost of an SP experiment is not independent of the SOC ratio, because multiple $(q)$ observations are taken on each subject. The total cost of the SP design is therefore $C_{\mathrm{SP}}=N_{\text {subj }}\left(S_{\text {cost }}+q O_{\text {cost }}\right)$. Recall that, for $\rho=$ .50 , a $2 \times 10 \mathrm{SP}$ design requires $N_{\text {subj }}=71$ subjects. If the SOC ratio is $3: 1$, the experiment costs $C_{\mathrm{SP}}=71[\$ 0.75+$ $10(\$ 0.25)]=71(\$ 3.25) \approx \$ 231$, and if the SOC ratio is $2: 1$, the experiment costs $71[\$ 0.67+10(\$ 0.33)]=71(\$ 3.97) \approx$ $\$ 282$. In either case, the SP design is more expensive than the CR design ( $\$ 128)$.

The cost of obtaining a single subject and a single observation in the experiment will not, of course, be exactly $\$ 1$ (the base dollar). Nevertheless, once we know the cost of the experiment in base dollars, we can scale the figure up or down accordingly. For example, suppose that the actual cost of obtaining a single subject and a single observation is $\$ 5$, not $\$ 1$. In this case, we simply multiply the cost solution obtained in base dollars by the appropriate multiple, $\$ 5 / \$ 1=5$. For the $2 \times 10 \mathrm{CR}$ design, the cost of the experiment would be $5(\$ 128)=\$ 640$; for the SP design $(\rho=.50)$, it would be $5(\$ 231)=\$ 1,155$ for a $3: 1$ SOC, and 5( $\$ 282)=\$ 1,410$ for a 2:1 SOC. The advantage of computing costs in base dollars is that it allows us to plot cost curves as a function of $\rho$ for various $p \times q$ SP designs and SOC ratios, read the cost in base dollars for our particular application, and then scale it up or down accordingly (using an appropriate cost multiplier). This allows one set of cost curves to be used, irrespective of the absolute cost of running a single subject and obtaining a single observation.

Figure 5 presents cost curves (in base dollars) for several $p \times q$ designs. The costs are computed on the basis of the sample sizes $\left(N_{\text {subj }}\right)$ plotted in Figure 3, which ensure power of 80 on $F_{A}$ for detecting a medium-size effect. The dashed horizontal line in each plot shows the cost of a CR design. As demonstrated above, the total cost for a $2 \times 10$ CR design is $\$ 128$. This cost is represented by the dashed line in the $2 \times 10$ plot of Figure 5 . Going from bottom to top, the solid lines show the cost curves for an SP design for each of the following SOC ratios: (1) $\infty: 1$ (\$1 and $\$ 0),(2) 3: 1$ ( $\$ 0.75$ and $\$ 0.25$ ), (3) $1: 1$ ( $\$ 0.50$ and $\$ 0.50)$, (4) $1: 3$ ( $\$ 0.25$ and $\$ 0.75$ ), and (5) $1: \infty(\$ 0$ and $\$ 1)$. To estimate the cost of an SP design, one simply selects the appropriate SOC curve (interpolating between them if necessary) and reads off the cost associated with a particular value of $\rho$. The 3:1 SOC curve for the $2 \times 10$ plot, for example, confirms the value obtained above for the cost of an SP design when $\rho=.50$ $(\approx \$ 231)$. The "true" cost of the experiment is determined by multiplying the cost in base dollars by an appropriate cost multiplier. For instance, if the actual cost of obtaining one subject and one observation is $\$ 14.25$, rather than $\$ 1$, then the cost in base dollars should be multiplied by 14.25 .

As we move from the bottom to top curve in any of the panels in Figure 5, the cost of obtaining a single subject is decreasing relative to the cost of obtaining a single ob- 

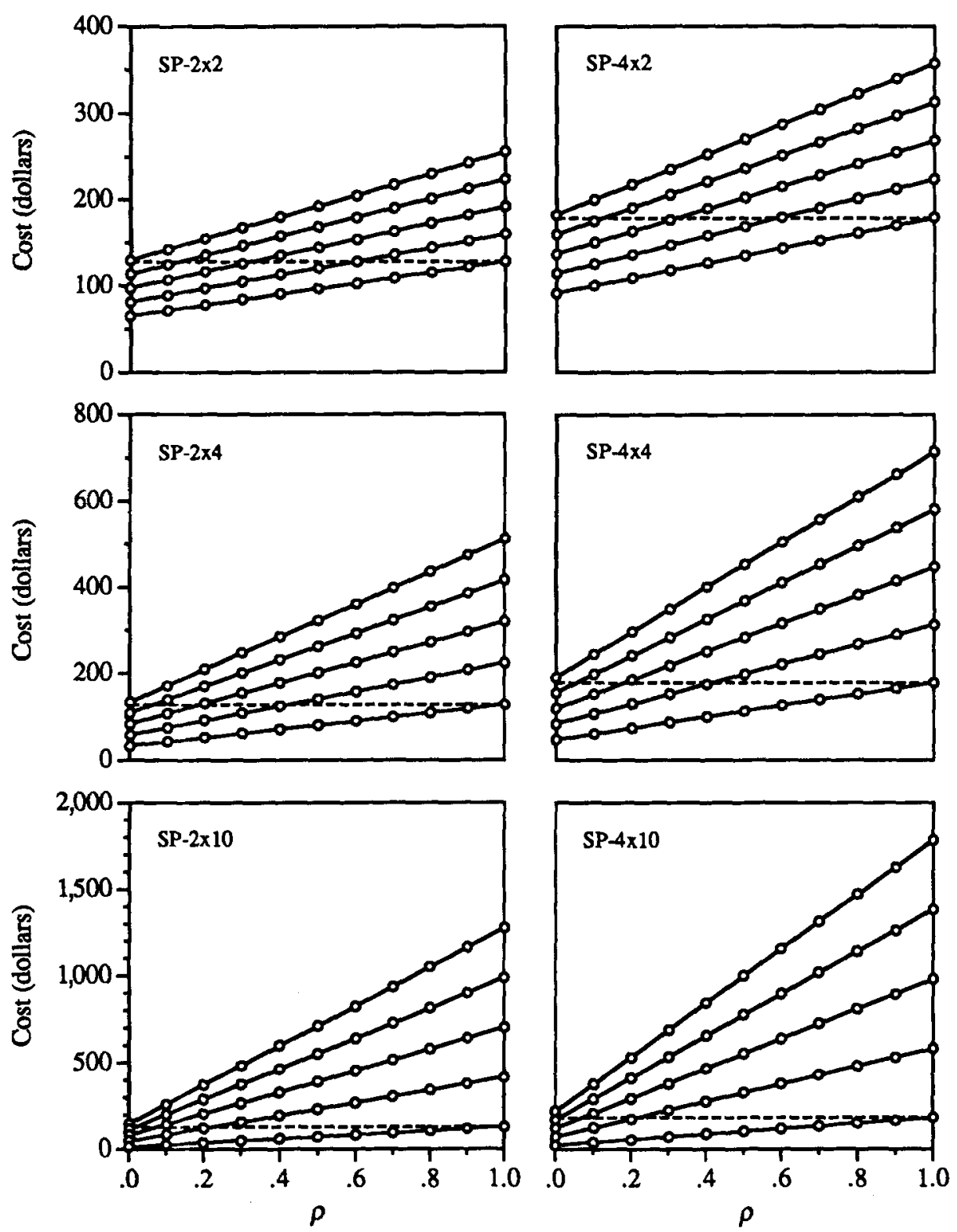

Figure 5. The total cost in "base" dollars required to achieve power of .80 for $F_{A}$ as a function of $\rho$ in $p \times q$ split-plot (SP) designs with correlated observations on factor $B$. The initial effect size is $f=$ .25 at $\rho=0$. From bottom to top, the five cost curves represent single subject and single observation costs, respectively, of (1) $\$ 1$ and $\$ 0$, (2) $\$ 0.75$ and $\$ 0.25$, (3) $\$ 0.50$ and $\$ 0.50,(4) \$ 0.25$ and $\$ 0.75$, and (5) \$0 and \$1. The dashed horizontal line shows the total cost for a completely randomized groups design where the single subject and single observation costs total to $\$ 1$.

servation. For the bottom curve, the cost of recruiting the subject accounts for the entire base dollar, and the cost of an observation is considered to be 0 . This gives an SOC ratio of $\infty: 1$; Figure 5 shows that the SP design is more efficient (less costly) than the CR design for all values of $\rho$ except $\rho=1$, where the two designs are equally efficient. For the top curve in each plot, the cost of recruiting a subject is considered to be 0 , and the cost of obtaining a single observation accounts for the entire base dollar (SOC $=1: \infty)$. When this is true, the CR de- sign is always more efficient than the SP design, as indicated by the fact that the $1: \infty$ cost curve never descends below the dashed horizontal line. For SOC ratios between these two extremes, the relative advantage of the SP design over the CR design depends on $\rho$. The crossover point, alluded to above, occurs where the cost curve for the SP design intersects the dashed horizontal line. At this point, the total cost of the experiment is the same for both designs. At lower values of $\rho$, the SP design costs less than the CR design; at higher values, the reverse is 
true. For designs with large $q$, such as the $2 \times 10$ and $4 \times 10$ designs, the cost of the SP design nearly always exceeds that of the CR design. Only $2 \times 2$ and $4 \times 2$ designs have a reasonable number of cases where the SP design outperforms the CR design, and, even here, this requires that the value of $\rho$ be fairly low. If the SOC ratio is 3:1, the crossover point for a $2 \times 2$ design falls at $\rho=$ .60 ; however, if the SOC ratio is $1: 1$, the crossover falls at $\rho=.33$. (For an SOC of $2: 1$, the crossover value is approximately midway between these two values.) If $q=4$, as in the $2 \times 4$ and $4 \times 4$ designs, the crossover value is only $\rho \approx .42$ for an SOC of 3:1, and the SP design will frequently be more expensive than the CR design.

\section{Cost Analysis and the SOC Ratio}

The SOC ratio explicitly captures the tradeoff in cost between obtaining subjects and observations in SP experimental designs. In research where the cost of subjects is extremely high (as in primate research, or research with specialized populations that are extremely difficult to reach), the SP design is very attractive because it keeps subject costs to a minimum. If the cost of obtaining observations is fairly low, then the resulting SOC ratio will be high, and the SP design is potentially competitive. For example, if the SOC ratio is $9: 1$, say, then the cost curves in Figure 5 suggest that the SP design may well be more efficient than a CR design, provided that the values of $\rho$ and $q$ are not too large. In contrast, the SP design will rarely be more efficient than a CR design if the cost and/ or effort required to recruit a subject is equal to or less than that required to obtain an observation (i.e., SOC $\leq 1$ ). Proper assessment of the SOC ratio is therefore paramount when attempting to decide which design to use. We consider next several factors that bear on the SOC ratio.

Consider an experiment to investigate the effects of eye movements on the magnitude of the Müller-Lyer illusion. The researcher plans to assign independent groups of subjects to either a scanning or a no-scanning eyemovement condition (factor $A$ ), and measure the strength of the illusion after $2,4,6$, or 8 min of inspection (factor $B$ ). The experimenter decides that factor $B$ can be either a within-groups or a between-groups factor. (If withingroups, each subject inspects the illusion for a total of $8 \mathrm{~min}$, and is measured at the 2-, 4-, 6-, and 8-min marks.) Since the subjects are volunteers and the data are collected using a computer program to display and measure the illusion, both the subject costs and the observation costs are relatively low. In this case, the choice of an SP versus a CR design may be dictated mainly by methodological, as opposed to statistical, considerations. If the experimenter believes that the underlying phenomenon of interest (illusion decrement) is best revealed when the subject has several opportunities to manipulate the measurement apparatus, then the SP design would be preferred. If it is thought that repeated testing confounds the results, then the CR design would be preferred (and this might well be true even if the design has less statistical power).

Continuing the preceding example, suppose that the experimenter intends to monitor the eye movements of the subjects as they scan or fixate the illusion. This is done by taking electrooculographic (EOG) recordings of the horizontal position of the eyes. In order to do this, the skin of each subject must be cleaned with alcohol, conducting gel applied, electrodes attached, and the EOG recorder carefully calibrated. The entire process takes 15-30 min, and recalibration checks are required at various points thereafter. This variation on the illusion experiment provides a good example of one in which the subject costs far exceed the observation costs. Note that subject preparation time is considered part of the subject cost because it is independent of the number of observations collected and it is part of the general overhead of recruiting and running subjects in the experiment. (Debriefing would also be considered a subject cost, for the same reason.) The SP design becomes very attractive in this instance because it answers the theoretical question of interest (illusion decrement as a function of scanning method) with potentially fewer subjects and therefore less "setup" time. Suppose, for example, that the experimenter decides that the cost of collecting an observation is essentially $\$ 0$ in comparison with the far greater costs of recruiting and preparing a subject. In this case, the SOC ratio approaches $\infty: 1$. The $2 \times 4$ panel in Figure 5 shows that the SP design will be less costly than the CR design for all values of $\rho$ except $\rho=1$. If the SOC ratio is very large, but not infinite, the crossover value for $\rho$ will be somewhat less than 1 , but not so low as to render the SP design less efficient than the CR design. Hence, in experiments having extraordinary subject acquisition and subject preparation costs, the SP design will generally be more efficient that the CR design.

Consider now an example of research where the observation costs are quite high in relation to the subject costs. A physiological psychologist wants to investigate the effects of nicotine on spatial memory. Rats are randomly assigned to either the experimental group or the control group and tested in a Morris water tank at 2, 4, 6 , or 8 days following initial training. For each test, the animal is placed in the water at a random location and allowed to swim to a submerged platform. The path the animal swims is video-recorded, and the total distance of the path from beginning to end serves as the dependent measure. Obtaining this measure is quite time consuming, because the video tape must be played back in slow motion and the path manually traced on an acetate sheet superimposed on the video monitor. A track wheel is then rolled over the path to measure the cumulative distance traveled. In this example, therefore, the effort required to obtain a single observation is quite considerable, and the SOC ratio would most likely be less than 1 (say, 1:2 or 1:3). Figure 5 makes it apparent that the $C R$ design will be more efficient than the SP design, except for very low values of $\rho .^{13}$

In addition to providing superior statistical power and/ or lower cost for many applications, CR designs have a number of other advantages. Since each subject serves in only one condition, there is no problem with order or carryover effects contaminating the results. CR designs also 
obviate the need to meet the more demanding statistical assumptions of repeated measures and SP designs (i.e., sphericity, multisample sphericity). Subject attrition is yet another advantage that CR designs have over SP designs. In the SP design, the loss of each subject results in the loss of many observations, and rerunning subjects is often more time consuming because of this. In addition, subject attrition is more likely to occur in a design that requires repeated measurements over time on the same subject than it is in a design requiring only one observation per subject. Finally, a last advantage of CR designs is that power analysis is simplified because only one error term is involved in the statistical analysis $\left(M S_{\mathrm{w} . c e l l}\right)$, and there is no requirement to estimate the value of $\rho$. Of course, when the nature of the theoretical question calls for repeated measures on factor $B$, as in studies that assess changes in performance over time and with repeated testing (subjects $\times$ trials designs), then the experimenter has no choice but to employ the SP design. In this event, the sample size analyses presented in Figure 3 will ensure acceptable power on factor $A$. The SP design may also prove feasible in situations where inadequate statistical power on factor $A$ is not an issue. For example, factor $A$ may not constitute a theoretically important variable, but it is included simply to extract systematic variance from the error term so as to yield a more powerful test. Gender is a between-groups variable that is included in many analyses for just this purpose. In this case, the SP design is really just a repeated measures design in which a grouping variable has been added to remove variance from the error term of the analysis. In this context, the reduced power on factor $A$ is not a liability, and the SP design provides considerable savings in the number of subjects required for the experiment, as well as increased power on factor $B$ and the $A B$ interaction. ${ }^{14}$

\section{Summary}

With respect to tests on the between-groups factor, the power, sample size, and cost analyses reported in this paper indicate that split-plot designs are most likely to exceed the performance of a completely randomized design when $\rho$ and $q$ are small and the SOC ratio is high. When this is the case, the savings in subjects tends to offset the requirement to collect more observations than would otherwise be needed by a completely randomized design. However, it is probably true that many researchers employ split-plot designs when these conditions are not being met. The examples in this paper illustrate the need for researchers to devote considerably more attention to issues surrounding power analysis and experimental design. Even though Cohen (1962) alerted researchers to the problem of inadequate power over 30 years ago, recent investigations by Sedlmeier and Gigerenzer (1989) and by Rossi (1990) indicate that little, if any, progress has been made in improving the power of studies published in psychological journals. The present analyses raise additional concerns with respect to the power of split-plot designs to detect effects in the between-groups factor. Paradoxically, the more effective the blocking of experimental units is in removing unwanted error variance for tests involving factor $B$ and the $A B$ interaction, the less effective the design will be in detecting effects due to factor $A$. As in most things, there is no "free lunch" in experimental design: The increased power of the tests on $B$ and the $A B$ interaction are purchased at the cost of dramatically reduced power on $A$. Consequently, if inferences about the main effects of $A$ constitute the theoretically interesting question to be addressed by the experiment, then either a split-plot design should be avoided or minimum sample size calculations should be performed to ensure adequate power for $F_{A}$.

\section{REFERENCES}

Bradley, D. R. (1988). DATASIM. Lewiston, ME: Desktop Press. BRADLEY, D. R. (1989). Computer simulation with DATASIM. Behavior Research Methods, Instruments, \& Computers, 21, 99-112.

Bradley, D. R., Russell, R. L., \& ReEVe, C. P. (1996). Statistical power in complex experimental designs. Behavior Research Methods, Instruments, \& Computers, 28, 319-326.

COHEN, J. (1962). The statistical power of abnormal-social psychological research: A review. Journal of Abnormal \& Social Psychology, 65 , 145-153.

COHEN, J. (1988). Statistical power analysis for the behavioral sciences (2nd ed.). Hillsdale, NJ: Erlbaum.

COOPER, H., \& FiNDLEY, M. (1982). Expected effect sizes: Estimates for statistical power analysis in social psychology. Personality \& Social Psychology Bulletin, 8, 168-173.

HaASE, R. F., WaEchter, D. M., \& Solomon, G. S. (1982). How significant is a significant difference? Average effect size of research in counseling psychology. Journal of Counseling Psychology, 29, 58-65.

KIRK, R. E. (1995). Experimental design: Procedures for the behavioral sciences (3rd ed.). Monterey, CA: Brooks/Cole.

Rossi, J. S. (1990). Statistical power of psychological research: What have we gained in 20 years? Journal of Consulting \& Clinical Psychology, 58, 646-656.

SEDLMEIER, P., \& GigerenZer, G. (1989). Do studies of statistical power have an effect on the power of studies? Psychological Bulletin, 105, 309-316.

Tixu, M. L. (1967). Tables of the power of the F-test. Journal of the American Statistical Association, 62, 525-539.

WINER, B. J. (1971). Statistical principles in experimental design (2nd ed.). New York: McGraw-Hill.

WINER, B. J., BROWN, D. R., \& MiCheLS, K. M. (1991). Statistical principles in experimental design (3rd ed.). New York: McGraw-Hill.

\section{NOTES}

1. These sample size values are verified by DATASIM 1.2 (Bradley, Russell, \& Reeve, 1996). The ESN command computes the sample size required to ensure a given level of power for various experimental designs. For a one-way design, the format of the command and an example based on the text are

ESN F $<k><$ design token $><\alpha>\{/ 1-\beta\}\{/ f\}$ \{precision\}

ESN F 2 S/T $.05 / .8 / .25$ \#\#.\#\#\#

The example computes the sample size required to ensure $1-\beta=.80$ $(\alpha=.05)$ for a medium-size effect $(f=.25)$ in a CR $(S / T)$ design with $k=2$ groups. The exact solution returned by DATASIM, $n=63.7656$, corresponds to an overall sample size of $N=127.5312$. (These values compare to $n=64$ and $N=128$ from Cohen's, 1988, tables, as noted in the text.) Most sample size tables display only integer values of $n$, whereas the ESN command computes and displays fractional solutions. 
This is necessary to allow more accurate determination of the overall sample size $(N)$ and to permit cross-checking by the ESP command. ESP is the reverse of ESN, in that it computes power given a particular sample size. The format of ESP and an example of a cross-check calculation are

ESP F $<k><$ design token $><\alpha>\{/ n\}\{/ f\}$ \{precision\}

ESP F 2 S/T $.05 / 63.7656 / .25$ \#.\#\#\#

Using the exact $n$ ensures that the returned power is $1-\beta=.8000$, rather than 8015 (returned for $n=64$ ). Yet another command, POWER, allows the user to compute power in terms of the noncentrality parameter, $\lambda$, rather than the effect size, $f$. Using the exact $N$ from above, we have $\lambda=N f^{2}=127.5312(.25)^{2}=7.9707$ and a denominator $d f$ of $v_{2}=$ $N-k=125.5312$. (This compares to $\lambda=8$ and $v_{2}=126$ as computed from $N=128$.) The format and an example of the POWER command are

POWER F $\left\langle v_{1}\right\rangle\left\langle v_{2}\right\rangle\langle\alpha\rangle\langle\lambda\rangle$

POWER F I 125.5312 .057 .9707

The returned value is exactly .8000 . Bradley et al. (1996) provide a complete overview of the ESP, ESN, and POWER commands, including how to generate and display power and sample size tables like those in Cohen (1988) and in other sources. These commands also compute power and sample size for $z, t, r$, and $\chi^{2}$. Finally, in addition to computing power and sample size, we note that DATASIM is a general-purpose data simulator and statistical analysis program (see Bradley, 1988, 1989).

2. For example, assume we have two experimental conditions: one that increases and one that decreases subjects' scores by an equal amount relative to a no-treatment control condition. In this case, the additional conditions that we add might represent multiple control groups (notreatment controls, placebo controls, etc.). Of course, there is no reason in general to expect that control groups will have means equal to the grand mean of the original two experimental conditions. We make this assumption here simply to facilitate the derivation of the attenuated effect size measure, $f^{\prime}$, discussed later in the text. Nevertheless, the conclusions we draw for Case 2 are valid even if the control group means are all above (or below) the experimental group means. The addition of the first control group might conceivably increase the overall effect size (since $\tau_{3} \neq 0$ ) and therefore power; however, as additional controls are added, the overall effect size will again decrease, as will power. This is because the grand mean will gradually shift in value toward that of the control groups, and, as they proliferate in number, the overall effect size measure will be attenuated by the many cases where $\tau_{j} \approx 0$. In fact, control groups are not really the central issue: Whenever an experimenter adds conditions to an experiment, all of which produce the same treatment effects, the effect size (and power) will decrease as $k$ goes up.

3 . Since $n=N / k$, this implies fractional values of $n$ for some values of $k$. This is not a problem for conducting power analysis, because there is nothing in the underlying mathematics that requires integer values of $n$ (or $v_{1}$ and $v_{2}$, for that matter). In practice, of course, the researcher would have to assign slightly different numbers of subjects to the $k$ conditions in order to maintain exactly $N=128$ overall.

4. Reallocating a fixed number of subjects $(N)$ to a greater number of conditions $(k)$ reduces the sample size per condition $(n)$, and this reduces the power of the test. This follows because the numerator of the $F$ ratio, $M S_{\mathrm{Bet}}$, is a function of $n$ times the variance of the means. Furthermore, although increasing the number of groups decreases $v_{2}$, the total $d f, v_{1}+v_{2}$, remains constant (Table 1). The reduction in power as $k$ increases is therefore the joint result of the conversion of denominator $d f$ $\left(v^{2}=N-k\right)$ to numerator $d f\left(v_{1}=k-1\right)$ and the reduction in sample size per condition $(n)$. To maintain constant power, either the conditions added to the experiment must produce treatment effects that exceed those already present or the overall sample size $(N)$ must be increased.

5 . Exact values of $n$ were computed by the ESN command of DATASIM 1.2 (see note 1) and multiplied by $k$ to obtain exact values of $N$. The exact values were then rounded to the nearest integer. Because of rounding error, the values of $n k$ computed from the entries in Table 1 sometimes differ slightly from the values of $N$ shown in the table.
6. Tiku's (1967) power tables display power as a function of the noncentrality parameter $\phi$. The value of $\phi$ may be determined from $\lambda$ or $f$ as foliows:

$$
\phi=\sqrt{\frac{\lambda}{v_{1}+1}}=f \sqrt{\frac{N}{v_{1}+1}} .
$$

7. Power was computed using the ESP command (see note 1). For a two-way design, the format of the command, and examples based on the CR and SP cases cited in the text, are

ESP F $\langle p \times q><$ design token $><$ effect token $><\alpha>$

$\{/ n\}\{/ f\} \quad$ precision $\}$

ESP F $2 \times 2$ S/AB A $.05 / 16 / .25000$ \#.\#\#\#

ESP F $2 \times 2$ B $\times$ S/A A $.05 / 16 / .20412 \#$ \#\#\#

The examples return power values for $F_{A}$ of .5032 and .3525 , respectively. The POWER command can also be employed to obtain the same solutions:

POWER F $\left\langle v_{1}\right\rangle\left\langle v_{2}\right\rangle\langle\alpha\rangle\langle\lambda\rangle$

POWER F $160 \quad .054 .000$

$\begin{array}{llllll}\text { POWER F } 1 & 30 & .05 & 2.667\end{array}$

As with the ESP command, the examples return power of .5032 and .3525 .

8. Note that SP power for the three effects at $\rho=0$ is slightly less than the CR power (.4904 vs. .5032 ), even though the effect size and noncentrality parameter are not modified at all when $\rho=0$. The lower power is due to the smaller error $d f$ associated with the SP design $\left(v_{2}=\right.$ $30)$ as opposed to the CR design $\left(v_{2}=60\right)$.

9. This is not an artifact due to the initially lower power of $F_{A}$ in the $2 \times 2$ plot of Figure 1 . We recomputed $F_{A}$ power for this case using $n=$ 30 , and we obtained power of .7682 and .4779 at $\rho=0$ and 1 , respectively. Comparing these with the values of .7750 and .1386 cited in the text for the $2 \times 10$ design, we see that, even when initial power (at $\rho=0$ ) is similar for both cases, the terminal power (at $p=1$ ) is much less in designs with large $q$.

10. This assumes that factor $B$ is a repeated measures factor. If the correlation is produced by using matched subjects, then no savings in subjects is achieved relative to a CR design.

11. The sample size values were computed using the ESN command described in note 1 . The format of the command for a two-way design is

ESN F $<p \times q><$ design token $><$ effect token $>\langle\alpha>$

$\{/ 1-\beta\}\{/ f\} \quad$ \{precision $\}$

We can illustrate this command for the examples cited in the text. The sample size required to achieve power of .80 for $f_{A(\mathrm{CR})}=.25$ in a $4 \times 4$ $\mathrm{CR}(S / A B)$ design is found by entering

\section{ESN F $4 \times 4$ S/AB A $.05 / .80 / .25$ \#\#\#\#\#\#\#}

The returned sample size is $n=11.1678$, and therefore $N_{\text {subj }}=N_{\text {obs }}=$ $n p q=178.6848$. For a $4 \times 4 \mathrm{SP}(B \times S / A)$ design with $\rho=.50$ and $f_{A}=$ $.25 / \sqrt{ }(1+3 \rho)=.158114$, the required sample size is found by entering

ESN F $4 \times 4$ BxS/A A .05 /.80/.158114 \#\#\#.\#\#

The returned value is $n=28.2526$, and therefore $N_{\text {subj }}=n p=113.0104$, and $N_{\text {obs }}=n p q=452.0416$. Note that the unrounded values of $n$ are used to compute exact values of $N_{\text {subj }}$ and $N_{\text {obs }}$. Since the sample size values reported in the text have been rounded to the nearest integer, $N_{\text {subj }}$ and $N_{\text {obs }}$ may not appear to be multiples (of $p$ and $p q$, respectively) of $n$. For example, computing $N_{\text {obs }}$ for the preceding example using the rounded value of $n$ gives $28(4)(4)=448$, whereas the exact solution is $28.2526(4)(4)=452.0416$.

12. The relative differences in sample size were computed using the absolute sample sizes plotted in Figure 3 for the $2 \times 2,2 \times 4$, and $2 \times 10$ designs.

13. If the researcher subsequently obtains video-processing software that can track the rat's path while swimming in the tank and compute the 
total distance traveled automatically, then the observation costs decrease relative to the subjects costs (SOC $>1$ ), and the SP design becomes potentially more attractive.

14. SP designs are often utilized in the field of biopsychology, where it is common to have an experimental condition in which rats undergo surgery to ablate or otherwise alter some region of the brain, and the effects of this surgery are then assessed in comparison to a sham-operated control group. Changes in the behavior of both groups are monitored over time by repeatedly testing the animals in a Morris water tank, shuttle box, tail-flick monitor, or similar apparatus. This approach could easily produce a $2 \times 10 \mathrm{SP}$ design like the one illustrated in the lower left panel of Figure 5 . It is clear that the SP design will nearly always be less efficient than the $C R$ design for this case. However, if the researcher's main interest is in assessing the interaction between $A$ and $B$, rather than the main effect of $A$, then the problem of attenuated power is less severe. This is because $F_{A B}$ is based on the same error variation,
$\sigma_{B \times S / A}^{2}=\sigma^{2}(1-\rho)$, as factor $B$ and therefore enjoys the same acceleration in power as $\rho$ goes from 0 to 1. Despite this reassuring fact, tests on interactions are generally less powerful than tests on main effects, sometimes dramatically so, due to the typically larger values of the numerator $d f$, where $v_{1}=(p-1)(q-1)$ for $F_{A B}$, as compared with $v_{1}=$ $(p-1)$ and $v_{1}=(q-1)$ for $F_{A}$ and $F_{B}$, respectively. Of course, if factor $A$ has only two levels, then no loss in the power of $F_{A B}$ occurs relative to $F_{B}$, and both tests benefit equally from the improved power due to correlated observations. For $p>2$, however, the power of $F_{A B}$ will be less than of $F_{B}$. As shown in Figure 1 for the $4 \times q$ designs, the power of $F_{A B}$ is substantially less than $F_{B}$. As a result, shifting the focus of the experimental hypothesis from the main effect of $A$ to the $A B$ interaction does not completely resolve the problem of diminished power in SP designs.

(Manuscript received August 13, 1996; revision accepted for publication May 30, 1997.) 